\title{
Evaluación de la ocupación del suelo con un sistema experto de evaluación de tierras y un SIG en la Zona de Especial Protección para las Aves «Encinares de los ríos Alberche y Cofio», Madrid
}

\section{Assessment of land cover with an expert system for land evaluation and GIS in Special Protection Area «Encinares de los ríos Alberche y Cofio», Madrid}

\author{
Javier Martínez-Vega* y Raúl Romero-Calcerrada**
}

\section{INTRODUCCIÓN}

Los países mediterráneos tienen una biodiversidad importante (Cowling et al., 1996; Blondel y Aronson, 1999). El manejo tradicional de las zonas rurales de estos países ha permitido que las zonas agrícolas y forestales mantengan una estrecha relación, incluso hoy en día, con su entorno. Sin embargo, los paisajes de Europa y del Mediterráneo se encuentran, cada vez, más amenazados por una intensificación de las actividades forestales y agrícolas (Stoate et al., 2001), así como por el desarrollo urbano, el turismo y las actividades recreativas al aire libre. Asimismo, el paulatino abandono de las actividades tradicionales, junto con la recesión económica y demográfica en algunas zonas rurales, está alterando el delicado equilibrio logrado por las actividades tradicionales (MacDonald et al., 2000). Como resultado, la diversidad biológica en

\footnotetext{
*Instituto de Economía, Geografía y Demografía, CCHS-CSIC (javier.martinez@cchs.csic.es).

${ }^{* * *}$ Escuela Superior de Ciencias Experimentales y Tecnología, Universidad Rey Juan Carlos, URJC (raul.romero.calcerrada@urjc.es).
} 
los ecosistemas mediterráneos está en declive (Caraveli, 2000; Pinto-Correia, 2000 y González Bernáldez, 1991).

Con el fin de preservar la diversidad biológica, la Unión Europea (UE) está promoviendo, desde hace unas décadas, una estrategia de conservación de la naturaleza basada en dos pilares: la Directiva Aves y la Directiva Hábitats. Las Zonas de Especial Protección para las Aves (ZEPA), designadas por la primera directiva, y los Lugares de Importancia Comunitaria (LIC) y Zonas de Especial Conservación (ZEC), designados por la segunda directiva, forman parte de la Red Natura 2000. Hasta enero de 2012, un total de 5.347 ZEPA y 22.594 Lugares de Importancia Comunitaria, han sido declarados en la UE, cubriendo una superficie terrestre de $517.340 \mathrm{~km}^{2}$ y $583.888 \mathrm{~km}^{2}$, respectivamente. A pesar de las reticencias de Francia para aportar espacios a la Red Natura 2000 como consecuencia de las restricciones de uso que pueden derivarse en su gestión, los países mediterráneos son los que aportan mayor biodiversidad a la Red. España tiene 593 ZEPA y 1.458 LIC terrestres. El conjunto de los lugares terrestres de la Red Natura 2000 en nuestro país ocupa el 27,2\% de la superficie nacional, un $10 \%$ más que la media de los 27 países miembros de la UE.

Además de su contribución a la conservación de la biodiversidad, estas zonas pueden constituirse como ejemplos de un desarrollo sostenible en que pueden coexistir usos tradicionales, con nuevos usos del suelo y con los servicios ambientales proporcionados por los ecosistemas agroforestales (Flores et al., 2008). La gestión sostenible de la tierra, tratando de preservar esta biodiversidad, requiere nuevas metodologías para la determinación de la capacidad general de usos del suelo y para su comparación con los usos actuales del suelo (RomeroCalcerrada, 2002), localizando, si los hubiere, los usos actuales que no están de acuerdo con los usos potenciales. Así pues, el método de Evaluación de Tierras se considera un marco válido para la identificación de los usos sostenibles de la tierra. Por tanto, es una herramienta eficaz en el ámbito de la planificación del uso del suelo, ayudando enormemente en el proceso de toma de decisiones (Bronsveld et al., 1994). De hecho, numerosos trabajos, que han seguido el marco metodológico de la FAO (1976), se han llevado a cabo en varios continentes con diferentes propósitos (Hommel, 1990; Zuviría y Valenzuela, 1994; Uboldi y Chuvieco, 1997). También se han desarrollado varios sistemas en forma de programas informáticos (Wood y Dent, 1983; Schultink, 1987; Rossiter y Van Wambeke, 1989; de la Rosa et al., 1992, 2004 y 2009), cuyos resultados pueden ser integrados en Sistemas de Información Geográfica (SIG).

Este trabajo tiene su sentido en el contexto actual de la incertidumbre que rodea a la planificación de algunas zonas de alto valor ecológico integradas en la Red Natura 2000. La falta de ideas claras en la ordenación de los recursos 
naturales, decisiones políticas contradictorias, conflictos de intereses entre distintos colectivos que representan a distintos sectores socioeconómicos son frecuentes en determinados lugares de esta red ecológica europea, amenazando la conservación de especies de fauna y flora protegidas y sus hábitats correspondientes. En Francia, se ha optado por la figura de contratos de gestión entre los propietarios y las autoridades con el fin de conciliar los objetivos de conservación de los recursos naturales y los de desarrollo socioeconómico. En la zona de estudio de este trabajo, Ecologistas en Acción (2006) ha denunciado el cambio del primer Plan de Ordenación de los Recursos Naturales (MartínezVega y Martín Lou, 2000) por otro más permisivo, realizado en 2005, y por el posterior Plan de Gestión del Espacio Protegido Red Natura 2000 denominado «Cuencas y Encinares de los ríos Alberche y Cofio», aprobado por el Decreto 36/2010 del Consejo de Gobierno de la Comunidad de Madrid. Mientras tanto, los municipios incluidos en esta área protegida han ido aprobando sus instrumentos de planeamiento urbanístico, ampliando y consolidando nuevos espacios de suelo urbano y urbanizable y se ha desdoblado una de las principales carreteras que atraviesan la zona (M-501), convirtiéndola en una vía de alta capacidad, obligando a adoptar una serie de medidas correctoras y compensatorias para reducir su impacto ambiental.

\section{OBjetivos}

Como se ha anticipado, el objetivo es aplicar un método de evaluación de la capacidad general de uso (CGU), integrado en un entorno SIG, en un territorio de alto valor ecológico: la Zona de Especial Protección para las Aves Encinares de los ríos Alberche y Cofio. La capacidad general de uso se compara con el uso actual de la tierra por medio de una tabulación cruzada. El objetivo es localizar las áreas críticas en las que los usos actuales no son adecuados para garantizar el desarrollo sostenible.

En este trabajo se intentará demostrar la eficiencia de la utilización de un SIG en la evaluación de tierras. Las rutinas del sistema experto de evaluación de tierras denominado MicroLEIS (de la Rosa et al., 1992, 2004 y 2009) se han integrado en un SIG ráster con el fin de que el proceso de evaluación y la representación cartográfica sean más prácticos. La configuración anexada entre ambos sistemas, que ha sido, tradicionalmente, la solución informática más extendida (Machín y Navas, 1995), se sustituye por una solución integrada más eficiente, en línea con otros trabajos más recientes (Navas et al., 2005; Lamelas, et al., 2009; Shahbazi y Jafarzadeh, 2010). 
ÁREA DE ESTUDIO

El área de estudio, llamado «Encinares de los ríos Alberche y Cofio», está situada en la Comunidad de Madrid (España), $40 \mathrm{~km}$ al suroeste de la capital (figura 1). Ocupa la cuenca media del río Alberche (afluente del Tajo). Tiene

FIGURA 1

ÁREA DE ESTUDIO

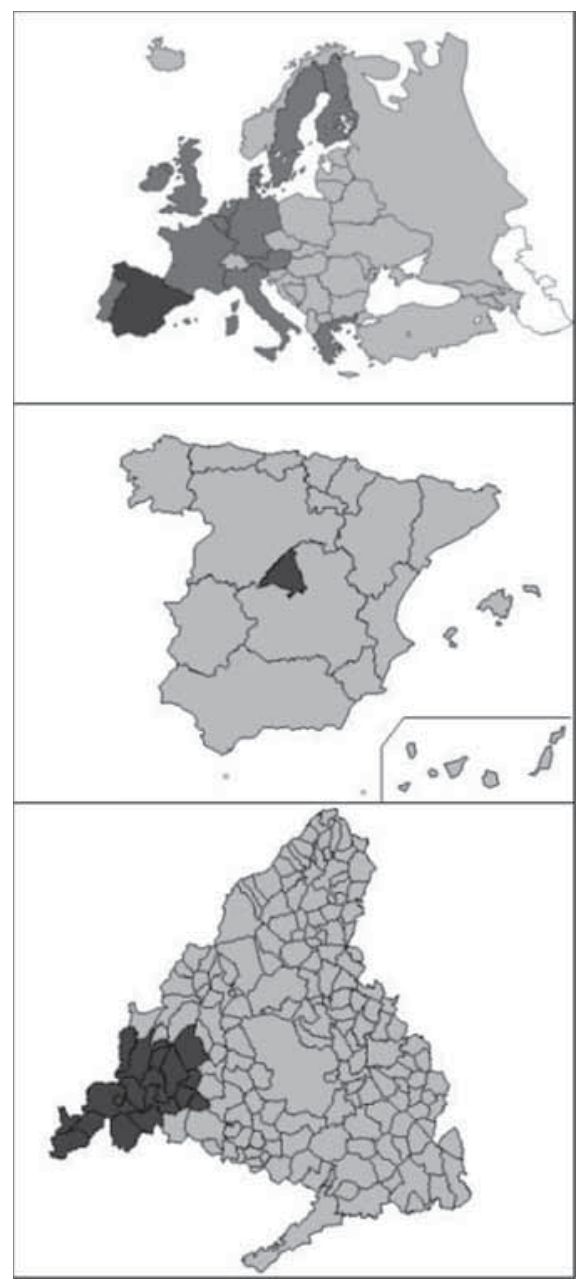

Fuente: elaboración propia.

Estudios Geográficos, Vol. LXXIII, 273, pp. 551-580, julio-diciembre 2012 ISSN: 0014-1496, eISSN: 1988-8546, doi: 10.3989/estgeogr.201219 
una superficie de $829,67 \mathrm{~km}^{2}$, afectando total o parcialmente a 19 municipios (tabla 1). De acuerdo con el censo de 2011, el área de estudio tiene una población de 66.277 habitantes y la densidad de población es baja $\left(86,48 \mathrm{hab} / \mathrm{km}^{2}\right)$ en comparación con la media de la Comunidad de Madrid $\left(807 \mathrm{hab} / \mathrm{km}^{2}\right)$. Sin embargo, algunos de estos municipios cuentan con recursos naturales de gran valor, como Pelayos de la Presa, que explican una importante presión demográfica $\left(331,40 \mathrm{hab} / \mathrm{km}^{2}\right)$ en su pequeño territorio. Además, la población aumenta, en gran medida durante los períodos de vacaciones, como resultado de la residencia secundaria y de la llegada de visitantes que vienen a practicar actividades de ocio al aire libre.

TABLA 1

INDICADORES DE LOS MUNICIPIOS INCLUIDOS EN EL ÁREA DE ESTUDIO

\begin{tabular}{lrrrrrrr}
\hline Nombre de municipios & $\begin{array}{c}\text { Superficie } \\
\text { municipal } \\
\text { (HA) }\end{array}$ & $\begin{array}{c}\text { Área } \\
\text { protegida } \\
(\mathrm{HA})\end{array}$ & $\begin{array}{c}\text { Área } \\
\text { protegida } \\
(\%)\end{array}$ & $\begin{array}{r}\text { Superficie } \\
\text { municipal } \\
\left(\mathrm{Km}^{2}\right)\end{array}$ & $\begin{array}{c}\text { Densidad } \\
(\text { hab//Km }\end{array}$ & $\begin{array}{c}\text { Población } \\
2011 \text { (Hab) }\end{array}$ & $\begin{array}{c}\mathrm{N}^{\circ} \\
\text { explotaciones } \\
\text { agrarias } \\
2009\end{array}$ \\
\hline Aldea del Fresno & 5.174 & 5.174 & 100.00 & 51,74 & 48,92 & 2.531 & 25 \\
Cadalso de los Vidrios & 4.760 & 3.823 & 80.32 & 47,60 & 61,05 & 2.906 & 123 \\
Cenicientos & 6.729 & 6.729 & 100.00 & 67,29 & 31,03 & 2.088 & 212 \\
Colmenar del Arroyo & 5.053 & 5.053 & 100.00 & 50,53 & 30,04 & 1.518 & 15 \\
Chapinería & 2.531 & 2.531 & 100.00 & 25,31 & 85,03 & 2.152 & 6 \\
Fresnedillas & 2.818 & 2.818 & 100.00 & 28,18 & 56,10 & 1.581 & 30 \\
Navalagamella & 7.585 & 7.585 & 100.00 & 75,85 & 31,21 & 2.367 & 42 \\
Navas del Rey & 5.102 & 5.102 & 100.00 & 51,02 & 52,57 & 2.682 & 43 \\
Pelayos de la Presa & 758 & 758 & 100.00 & 7,58 & 331,40 & 2.512 & 8 \\
Quijorna & 2.570 & 954 & 37.11 & 25,70 & 117,12 & 3.010 & 12 \\
Robledo de Chavela & 9.300 & 7.565 & 81,35 & 93 & 42,91 & 3.991 & 43 \\
Rozas de Puerto Real & 3.020 & 2.466 & 81,66 & 30,20 & 15,43 & 466 & 37 \\
San Martín de Valdeiglesias & 11.550 & 10.069 & 87.17 & 115,5 & 71,31 & 8.236 & 238 \\
Sevilla la Nueva & 2.486 & 2.486 & 100.00 & 24,86 & 354,30 & 8.808 & 8 \\
Valdemaqueda & 5.172 & 5.172 & 100.00 & 51,72 & 16,65 & 861 & 4 \\
Valdemorillo & 9.370 & 1.779 & 18.99 & 93,70 & 123,21 & 11.545 & 52 \\
Villa del Prado & 7.840 & 7.361 & 93.89 & 78,40 & 82,35 & 6.456 & 246 \\
Villamantilla & 2.396 & 2.396 & 100.00 & 23,96 & 45,45 & 1.089 & 14 \\
Villanueva de Perales & 3.147 & 3.147 & 100.00 & 31,47 & 46,97 & 1.478 & 14 \\
\hline
\end{tabular}

$\begin{array}{llllllll}\text { TOTAL ZEPA } & 97.361 & 82.968 & 85.14 & 973,61 & 86,48 & 66.277 & 1.172\end{array}$

Fuentes: elaboración propia a partir de datos del Instituto de Estadística de la Comunidad de Madrid (2012) y el Censo Agrario de España (2009). 
Esta zona es de gran interés para la conservación de la naturaleza y, especialmente, para las aves (Romero-Calcerrada, 2000). Contiene hábitats que son el soporte de una fauna muy rica y diversa (Consejería de Medio Ambiente y Desarrollo Regional de la Comunidad de Madrid, 1998 y Álvarez y Díaz, 1997). El número de especies de aves en la zona asciende a más de 150. El estado de conservación de las comunidades biológicas existentes es excelente y, además, la importancia de algunas especies amenazadas es extraordinaria. Especialmente destacable es la presencia de fauna en serio peligro de extinción a escala nacional y europea, incluyendo una especie endémica, el águila imperial ibérica (Aquila adalberti) (Garzón et al., 1984 y Veiga et al., 1984), el buitre negro (Aegypius monachus), el buitre leonado (Gyps fulvus), el búho real (Bubo bubo), la cigüeña negra (Ciconia nigra), el lince ibérico (Lynx pardina) (Aldama, 1996 y García Perera, 2000) y la nutria (Lutra lutra) (García González, 1998).

La flora también es un recurso muy valioso. El bosque mediterráneo es dominante en esta zona. Las principales especies presentes son (Martínez-Vega y Martín-Lou, 2000) el pino piñonero (Pinus pinea) y el pino marítimo (Pinus pinaster), la encina (Quercus ilex), el roble (Quercus pyrenaica), el castaño (Castanea sativa) y las formaciones galería de álamo, sauce, fresno y alisos. Según el Ministerio de Medio Ambiente, 20 hábitats, de los que figuran en el anexo 1 de la Directiva Hábitats, están presentes en el área de estudio, ocupando una superficie de más de 30.000 ha ( $37 \%$ de la zona de estudio). Los estanques temporales mediterráneos, pseudoestepas con gramíneas anuales del Thero-Brachypodietea y bosques fluviales de Alnus glutinosa y Fraxinus excelsior son hábitats prioritarios en el área de estudio. Como resultado de esta riqueza biológica y paisajística, principalmente en aves, esta zona fue declarada ZEPA por el Gobierno regional en 1990. Adicionalmente, buena parte de su territorio posee una elevada calidad visual del paisaje. También es una zona de gran interés desde el punto de vista natural, porque presta una importante función como conector biofísico entre las sierras de Malagón y la de Guadarrama, uniendo otros espacios naturales protegidos.

A pesar de su valioso patrimonio natural, han ocurrido negativos cambios de uso del suelo desde 1982 (Gallardo y Martínez-Vega, 2012; Pérez-Hugalde et al., 2011), y aún antes, debido a su proximidad al área metropolitana de Madrid, con consecuencias adversas para la conservación de los recursos naturales. Entre estos cambios, podemos destacar la proliferación de urbanizaciones de vivienda secundaria, el abandono de los usos tradicionales (Romero-Calcerrada y Perry, 2004) y las alteraciones del paisaje (Romero-Calcerrada y Perry, 2002). La interfaz urbano-forestal está estrechamente relacionada con la ocurrencia de incendios forestales en el área de estudio (Martínez-Vega et al., 
2003; Chuvieco et al., 2010 y Romero-Calcerrada et al. 2010). La proximidad de la ZEPA a Madrid atrae a un gran número de visitantes que realizan actividades al aire libre en dos embalses del río Alberche (San Juan y Picadas) y en algunos tramos del río Alberche. Tal concentración de visitantes provoca un fuerte impacto ambiental — contaminación de agua como resultado de los combustibles empleados por las embarcaciones, eutrofización, compactación del suelo, daños a la vegetación, vertidos de residuos sólidos, ruidos, etc.- . Como se ha anticipado, otro impacto significativo ha sido el desdoblamiento de una carretera que atraviesa áreas de alto valor ecológico, incrementando el ancho de su calzada y el volumen de tráfico que soporta (Ecologistas en Acción, 2006). Su construcción ha incrementado la fragilidad del paisaje incluido en su cuenca visual y ha dificultado la conectividad de ciertos corredores ecológicos. Mientras tanto, la presión del desarrollo urbano ha exigido nuevas tierras para la construcción de urbanizaciones de vivienda secundaria en las zonas con mayor belleza escénica. Asimismo, existen algunas urbanizaciones ilegales sobre suelo no urbanizable de alto valor ambiental, instalaciones industriales y ganaderas intensivas y líneas eléctricas de alta y media tensión.

\section{MATERIAL Y MÉTODOS}

Se han utilizado diversos materiales y fuentes de información geográfica para esta investigación. Se ha elaborado un Modelo Digital de Elevaciones (MDE) a partir de la información proporcionada por el Servicio Cartográfico Regional. El modelo se ha calculado a partir de las coordenadas $\mathrm{x}, \mathrm{y}, \mathrm{z}$ de una serie de entidades topográficas relevantes del área de estudio y de su entorno geográfico: cotas, vértices geodésicos y curvas de nivel equidistantes cada 20 $\mathrm{m}$. Incorpora los puntos de ruptura de pistas y otras entidades planas como superficies de agua. Este es un método muy selectivo, dado que sólo las características más destacadas del terreno están, explícita o implícitamente, recogidas. El resultado es un modelo con una estructura TIN (Peucker et al., 1978) en la que los triángulos de Delaunay (Kidner y Jones, 1991) se determinan a partir de los polígonos de Thiessen. Este modelo ha sido convertido en un MDE ráster, con un tamaño de celda de $20 \mathrm{~m}$ de lado.

La distribución espacial de la precipitación total anual, el total de la evapotranspiración potencial anual y el riesgo de heladas se ha llevado a cabo mediante la interpolación de los datos de 21 estaciones meteorológicas en el área de estudio y en su entorno geográfico. Estos datos han sido proporcionados por el Instituto Nacional de Meteorología. 
El mapa de suelos se ha derivado, mediante trabajo de campo y la interpretación de fotografías aéreas estereoscópicas de un vuelo de 1995 sobre la región de Madrid, en color natural, a escala 1:18.000. Se han delimitado diecinueve unidades cartográficas de suelos, siguiendo la clasificación de la FAO-UNESCO (1989).

Asimismo, se ha utilizado un mapa de vegetación y usos del suelo, elaborado en 1997 por el Departamento de Medio Ambiente de la Comunidad de Madrid a partir de trabajo de campo y la interpretación de fotografías aéreas. Este mapa está en formato vectorial, a una escala de 1:50.000, y se ha dividido en 52 categorías diferentes. Éstas describen el dominio y la asociación de las formaciones vegetales así como su estructura vertical y horizontal. No se ha utilizado ningún otro mapa de ocupación y usos del suelo más reciente (CORINE-Land Cover 2000 o 2006; Romero-Calcerrada y Perry, 2002 y 2004) por ser éstos de escala más pequeña y, por tanto, menos detallada o por disponer de una leyenda menos desagregada.

Buena parte de los datos originales provienen de agencias gubernamentales y cumplen con los estándares de calidad requeridos. Otros productos, generados por nuestro grupo de trabajo, han sido verificados por medio de análisis espacial (por ejemplo, la interpolación de las alturas del MDE). Siguiendo otros trabajos (Chuvieco et al., 1991), un muestreo aleatorio no alineado se ha llevado a cabo, con un nivel de confianza del $95 \%$. Un total de 830 puntos han sido seleccionados en la matriz de celdas de $1 \mathrm{~km}^{2}$. Una matriz de confusión (Aronoff, 1989) se ha derivado después de haber comprobado la elevación de cada punto con la ayuda de mapas topográficos de escala más grande (1:25.000). En base a esta matriz de confusión, se estima que el MDE generado tiene una fiabilidad global del $96 \%$, con un error promedio de 9,5 m.

Se han aplicado funciones estándar de los SIG para la depuración y la corrección de errores (Chrisman, 1989) en la representación vectorial de los datos digitalizados (por ejemplo, mapa de suelos). Para la conversión de los mapas vectoriales a formato ráster (mapas de suelos y de usos del suelo) se ha utilizado un tamaño de celda pequeño $(20 \times 20 \mathrm{~m})$, con el fin de minimizar los errores derivados de la rasterización. Cuanto más corta sea la longitud lateral de la celda, menor es el error estándar resultante de la rasterización de un mapa vectorial (Frolov y Maling, 1969).

Metodológicamente, se ha utilizado un sistema de Evaluación de Tierras, basado en el esquema de la FAO (1976) y en el sistema experto MicroLEIS (de la Rosa et al., 1992; 2004; 2009; Navas et al., 2005; Lamelas, et al., 2009; Shahbazi y Jafarzadeh, 2010). MicroLEIS es un sistema informático de evaluación de tierras desarrollado para determinar el uso óptimo de las tierras agrí- 
colas y forestales en condiciones mediterráneas. Es un método empírico que tiene en cuenta un conjunto de variables biofísicas y aplica un enfoque multiescalar. Se emplean métodos cualitativos, a escala de reconocimiento, y cuantitativos (Verheye, 1987), a escala de detalle.

MicroLEIS contiene un sistema de menús y se estructura en tres secciones principales:

- Inf\&Kno: se utiliza para llevar a cabo un inventario de los recursos rurales y transferir datos básicos. Una base de datos de suelos (SDBm) se incluye para la organización, almacenamiento y análisis de la información básica que se deriva de los datos morfológicos y analíticos sobre los perfiles de suelos. SDBm facilita la transferencia de datos a los sistemas de información territorial y de evaluación de los SIG. Una base de datos agroclimáticos (CDBm) también se incluye para la evaluación de tierras.

- Pro\&Eco: este módulo se utiliza para evaluar la calidad de la tierra y la construcción de los modelos diseñados para diferentes aspectos: capacidad de uso general, la deficiencia bioclimática, aptitud agrícola. Estos modelos siguen los criterios de evaluación de tierras de la FAO (1976) y las adaptaciones hechas por la UE. Los cálculos son empíricos y son formulados y calibrados usando el conocimiento experto.

- Ero\&Con: este módulo evalúa la vulnerabilidad de la tierra y construye modelos sobre los riesgos de degradación.

Los procesos que se han seguido son los del modelo Cervatana, que se incluye en el módulo Pro\&Eco. El propósito del modelo Cervatana es definir la capacidad general de uso de las tierras (CGU), entendida como la aptitud de los suelos para una serie de usos. Estos usos se enumeran en un orden decreciente según su intensidad, desde la agricultura y la ganadería hasta la silvicultura y las funciones ambientales de protección y de conservación. Se supone que tienen lugar en el territorio de una manera continua sin empobrecer el suelo (FAO, 1976 y USDA, 1961).

El modelo de CGU es el resultado de la síntesis de cuatro factores: pendiente, suelo, riesgo de erosión y deficiencia bioclimática. Cada uno de estos factores se ha obtenido, a su vez, mediante la agrupación de los valores de doce variables biofísicas. Cada variable se ha obtenido de fuentes distintas (MDE, un mapa de suelos, un mapa de vegetación y usos del suelo y una base de datos climáticos). Como se ha indicado, ciertas variables han sido convertidas de formato vectorial a ráster, usando un tamaño común de celda de $20 \mathrm{x}$ $20 \mathrm{~m}$. Cada celda sirve como unidad geográfica de referencia, considerándose homogénea desde el punto de vista biofísico. 
Un primer paso en la aplicación de esta metodología consiste en convertir los valores de cada una de las variables en una escala común de medida. Los valores iniciales de cada variable se han reclasificado en una escala ordinal (de la Rosa et al., 1992), que va desde el valor 1, cuando no existen limitaciones, hasta 3 o 4, cuando coexisten las limitaciones máximas. Cada factor integra los valores ordinales de todas las variables que se relacionan con ellos.

\section{El factor pendiente $(\mathrm{t})$}

Este factor se obtuvo del MDE. Para definir la pendiente, el modelo examina las diferencias de altitud entre cada píxel central y la de cada uno de sus vecinos, calculando el gradiente de la pendiente máxima, en porcentaje, en las dos direcciones básicas: norte-sur y este-oeste (Intergraph, 1994). La ecuación utilizada es:

$$
s=\sqrt{ }\left(p^{2}+q^{2}\right)
$$

Donde:

$\mathrm{s}=$ la pendiente máxima del píxel central.

$\mathrm{p}=$ la pendiente en la dirección este-oeste.

$\mathrm{q}=$ la pendiente en la dirección norte-sur.

Según Skydmore (1989), este es uno de los métodos más adecuados para calcular la pendiente. Los valores de la pendiente se han agrupado en cuatro intervalos: inferior al 7\%, del 7 al 15\%, del 15 al 30\% y superior al 30\%. De acuerdo a su influencia sobre el drenaje, la erosión y la mecanización, los cuatro intervalos han sido reclasificados de acuerdo con los valores de la figura 2 , que expresa la limitación de este factor sobre la CGU utilizando una escala ordinal.

FIGURA 2

\section{FACTOR PENDIENTE}

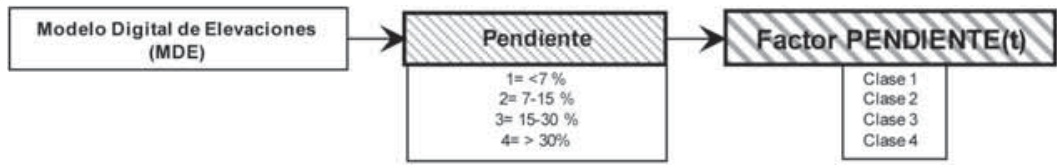

Fuente: elaboración propia. 


\section{Factor riesgo de erosión (r)}

Integra cuatro variables: la pendiente, la densidad de la vegetación, la erosividad de las lluvias y la erodibilidad del suelo (figura 3).

- Pendiente: evidentemente, cuanto mayor sea la pendiente, mayor es el riesgo de erosión. Las celdas del modelo digital de pendientes se han reclasificado en tres grupos: el primero contiene aquellos píxeles que tienen pendientes inferiores a $15 \%$; el segundo, los que poseen inclinaciones entre 15 y $30 \%$ y en el último se agrupan las celdas con valores superiores al $30 \%$.

- Densidad de la vegetación: como es sabido, la vegetación protege el suelo evitando así la desintegración y el desprendimiento de partículas minerales. El mapa de vegetación se ha reclasificado de acuerdo con el porcentaje de cobertura de cada una de sus categorías o la fracción de superficie cubierta por cada tipo de vegetación. En el caso de la vegetación estacional o de los cultivos se tiene en cuenta el número de meses por año durante el cual se priva al suelo de una cubierta protectora.

- La erosividad producida por la lluvia (valor R de la ecuación USLE) depende de la intensidad de ésta y de su impacto sobre la superficie, que a su vez dependen de las características físicas de la precipitación. Las iso-

FIGURA 3

FACTOR RIESGO DE EROSIÓN

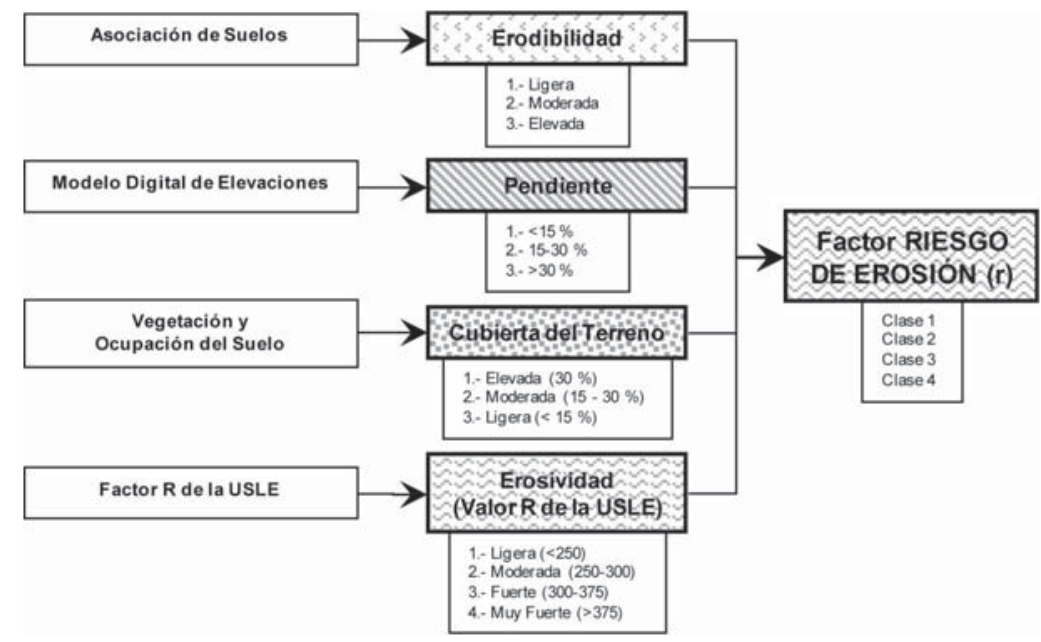

Fuente: elaboración propia. 
líneas que expresan los valores de erosividad en el área de estudio se han extraído de una publicación específica (ICONA, 1988). Estos valores de erosividad, obtenidos mediante interpolación, se han reclasificado teniendo en cuenta los intervalos definidos en la figura 3.

- La erodibilidad representa la vulnerabilidad o resistencia del suelo a la desintegración y desprendimiento de partículas en su superficie. Esta variable se ha obtenido mediante la reclasificación del mapa de suelos de acuerdo al factor $\mathrm{K}$ de la ecuación USLE. Los valores han sido agrupados, posteriormente, en un índice cualitativo.

\section{Factor suelo (1)}

Integra cinco variables. Cada una de ellas se ha obtenido mediante la reclasificación del mapa de suelos (figura 4).

- Profundidad útil, expresada en centímetros. Se considera como la parte del suelo que es fácilmente penetrada por las raíces. En virtud de esta variable, los suelos se han reclasificado en 4 grupos, de acuerdo a los intervalos señalados en la figura 4.

- La textura se entiende como la proporción de arena, limo y arcilla. Todos los suelos que tienen una textura media o pesada se han reclasificado como 1 , mientras que a los suelos de textura ligera se les ha asignado el valor 2 .

- Pedregosidad o rocosidad se refiere al porcentaje de la superficie cubierta por fragmentos gruesos y/o rocas. Se han diferenciado tres grupos (figura 4).

- El drenaje se considera como la eliminación natural de agua del suelo. Se distinguen varios niveles según la velocidad o facilidad con que esto ocurre. Un valor de 1 se asigna a los suelos con buen drenaje, valor 2 para aquellos con drenaje moderado y los que tienen drenaje deficiente - demasiado rápido o demasiado lento- son reclasificados en un tercer grupo.

- Salinidad, se refiere a la concentración de sales solubles en el perfil del suelo. Se expresa en $\mathrm{d} S / \mathrm{m}(\mathrm{mmhos} / \mathrm{cm})$. Los suelos son recategorizados de acuerdo con los intervalos de la figura 4.

\section{Factor deficiencia bioclimática (b)}

Este factor se ha derivado de la combinación de dos variables: deficiencia hídrica y el riesgo de heladas (figura 5). 
FIGURA 4

FACTOR SUELO

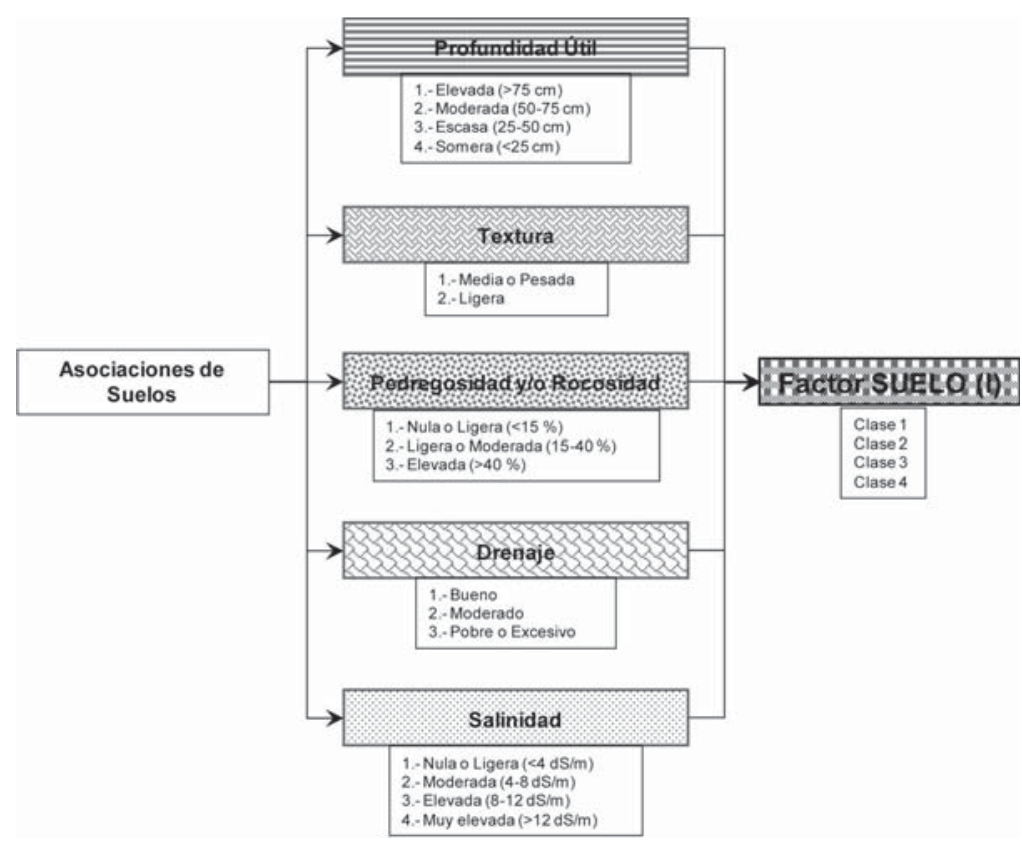

Fuente: elaboración propia.

FIGURA 5

FACTOR DEFICIENCIA BIOCLIMÁTICA

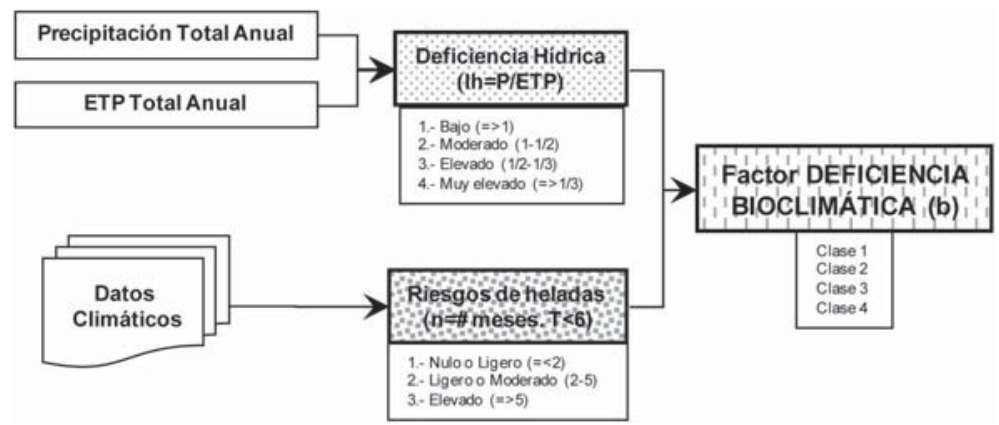

Fuente: elaboración propia. 
La deficiencia hídrica se ha obtenido dividiendo la precipitación total anual (en $\mathrm{mm}$ ) por la evapotranspiración potencial anual (en $\mathrm{mm}$ ), siendo calculada, ésta última, según el método de Thornthwaite (1948).

$$
D H=P / E T P
$$

Donde:

DH = Deficiencia hídrica.

$\mathrm{P}=$ Precipitación total anual $(\mathrm{mm})$.

ETP $=$ evapotranspiración potencial $(\mathrm{mm})$.

Para modelar cartográficamente la precipitación anual y la evapotranspiración potencial, han sido interpolados espacialmente los datos de las estaciones meteorológicas, considerando su correlación con otras variables y aplicando las siguientes ecuaciones (Romero-Calcerrada y Martínez-Vega, 2001):

$$
\begin{gathered}
P=(0.420395 * E)-(14.731575 * O)+(7,531799 * G P)-(0,001592 * \\
L O)-(0,001127 * L A)+5981,83223 \\
\text { ETP }=(-0.032109 * P)+(40,137815 * T M)+(0,164626 * \text { TMmax })+ \\
(0,005474 * E)+(1,560118 * O)+117.25319 \\
T M=(-0,008352 E *)+(0,52282 * O)+(0,052598 * G P)- \\
(0,00000437813 * L O)-(0.000000458646 * L A)+21,033924 \\
T M \operatorname{TMax}=(-0,006078 * E)+(0,410993 * O)-(0,076623 * G P)+ \\
(0,00000510739 * L O)-(0,0000511688 * L A)+249,478867
\end{gathered}
$$

Donde:

$\mathrm{P}=$ Precipitación $(\mathrm{mm})$.

ETP = Evapotranspiración Potencial $(\mathrm{mm})$.

$\mathrm{TM}=$ Temperatura media anual $\left({ }^{\circ} \mathrm{C}\right)$.

TMmax $=$ La media anual de temperatura máxima $\left({ }^{\circ} \mathrm{C}\right)$.

$\mathrm{E}=$ Elevación $(\mathrm{m})$.

$\mathrm{O}=$ Orientación $\left(^{\circ}\right)$.

$\mathrm{GP}=$ Gradiente de la Pendiente (\%).

$\mathrm{LO}=$ Longitud (UTM).

$\mathrm{LA}=$ Latitud $(\mathrm{UTM})$.

El cociente resultante (DH) se ha reclasificado de acuerdo con la tabla de conversión de la figura 5. 
El riesgo de helada se ha calculado según el número de meses con una temperatura media mínima por debajo de $6^{\circ} \mathrm{C}$. Cada celda está recategorizada según el número de meses con riesgo de heladas.

\section{Capacidad General de Uso}

Los cuatro factores limitantes - pendiente (t), riesgo de erosión $(\mathrm{r})$, suelo (l) y deficiencia bioclimática (b) — están integrados en el mapa de CGU (figura 6). De esta manera, se tiene en cuenta un amplio abanico de características, cualidades y limitaciones ambientales de las tierras.

A pesar de las ventajas de los métodos de combinación lineal ponderada (Eastman, 2000), que obtiene una compensación total entre los factores que forman parte de la combinación, y del método OWA (Eastman, 2000), que ofrece la posibilidad de controlar el nivel de compensación entre los factores, la regla de decisión, utilizada en este trabajo, para la integración de las variables y de los factores se ha basado en técnicas no compensatorias. Los valores má-

\section{FIGURA 6}

FLUJO DE TRABAJO PARA LA EVALUACIÓN DE TIERRAS

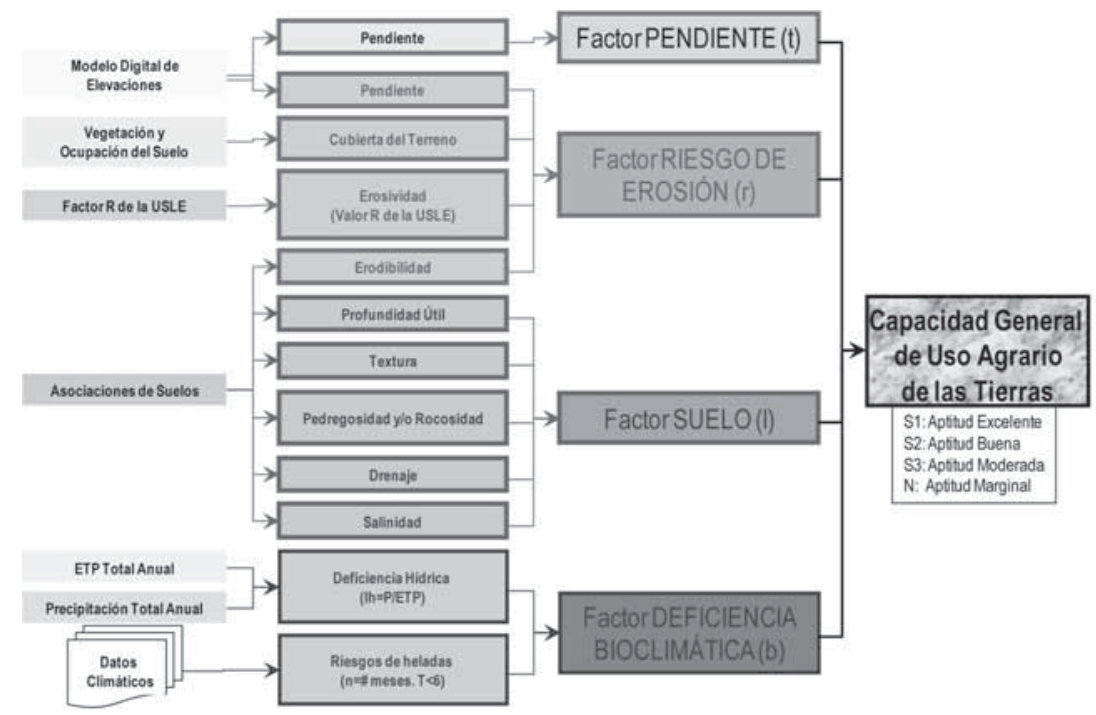

Fuente: elaboración propia. 
ximos de las variables y factores limitantes son dominantes porque establecen restricciones físicas importantes para un aprovechamiento agroforestal intensivo y continuado de las tierras. Estas limitaciones o carencias biofísicas de las tierras no pueden ser compensadas por valores positivos de otros factores. No son intercambiables.

La siguiente rutina ha sido programada en el módulo ráster del software MGE de Intergraph:

$$
\begin{gathered}
\text { int } \mathrm{M} ; \\
\mathrm{M}:=\operatorname{MAX}\{\mathrm{i} 1, \mathrm{i} 2, \mathrm{i} 3, \mathrm{i} 4\} ; \\
\text { ol: }=\mathrm{M} ;
\end{gathered}
$$

Donde $\mathrm{M}$ es una variable entera que toma el valor máximo de los 4 factores de entrada (i1 = factor pendiente; $\mathrm{i} 2$ = factor riesgo de erosión; $\mathrm{i} 3$ = factor suelo; i 4 = factor de deficiencia bioclimática). $\mathrm{M}$ es el nuevo valor de salida (ol) para ser asignado al píxel.

De esta manera, cada píxel tiene un valor final de CGU comprendido entre 1 (mayor capacidad general de uso y menores limitaciones) y 4 (menor capacidad general de uso como consecuencia de mayores restricciones biofísicas). La CGU puede equipararse a la aptitud que tienen las tierras para soportar una gama de usos agrosilvopastorales de mayor a menor intensidad.

La figura 7 muestra un ejemplo de las posibles combinaciones de los factores en nueve unidades de tierra o píxeles imaginarios. Cada celda del mapa final (CGU) toma el valor máximo de las cuatro factores integrados (pendiente «t», riesgo de erosión «r», suelo «l» y deficiencia bioclimática «b»). Así, por ejemplo, el píxel superior izquierdo de la matriz no tiene ninguna limitación de uso derivada de la pendiente, el riesgo de erosión o el suelo. Sin embargo, sí tiene un déficit bioclimático moderado (valor 2), como resultado de la escasez de agua o del riesgo de heladas en esa unidad tierra. En el mapa de CGU, esta celda tiene un valor de 2 . El valor 1 de este mapa final es equivalente a la clase S1 de la FAO; indica una excelente CGU. El valor 2 (S2) muestra una buena CGU, el valor 3 (S3) una capacidad de uso moderada y el valor 4 (clase N) una capacidad marginal.

Cada dígito de la clase va seguido de uno o varios identificadores (en letras minúsculas) de la subclase o subclases a las que pertenece cada píxel. Estos identificadores indican el factor o factores que limitan, negativamente, la CGU de esa unidad tierra. Si el factor limitante es la topografía, la letra t acompaña a la clase. Si el factor limitante es la capacidad de riesgo de erosión, la letra que acompaña a la clase es $\mathrm{r}$. Si el elemento limitante es el suelo, la letra adjunta es l. Por último, si la deficiencia bioclimática es la causa de la limitación, 
la letra b es añadida. En el caso de que varios factores limitantes estén presentes a la vez, una celda puede ser designada por una clase y varias subclases, colocadas éstas en orden de mayor a menor limitación.

En la figura 7, el píxel de la fila central y columna derecha pertenece a la clase S3 (valor 3) y t, l y b son los factores limitantes. Su atributo es por lo tanto S3tlb, por lo que esta unidad tierra tiene una moderada CGU y sus principales factores limitantes son la topografía, el suelo y la deficiencia bioclimática.

\section{FIGURA 7}

\section{UN EJEMPLO DE COMBINACIÓN DE FACTORES}

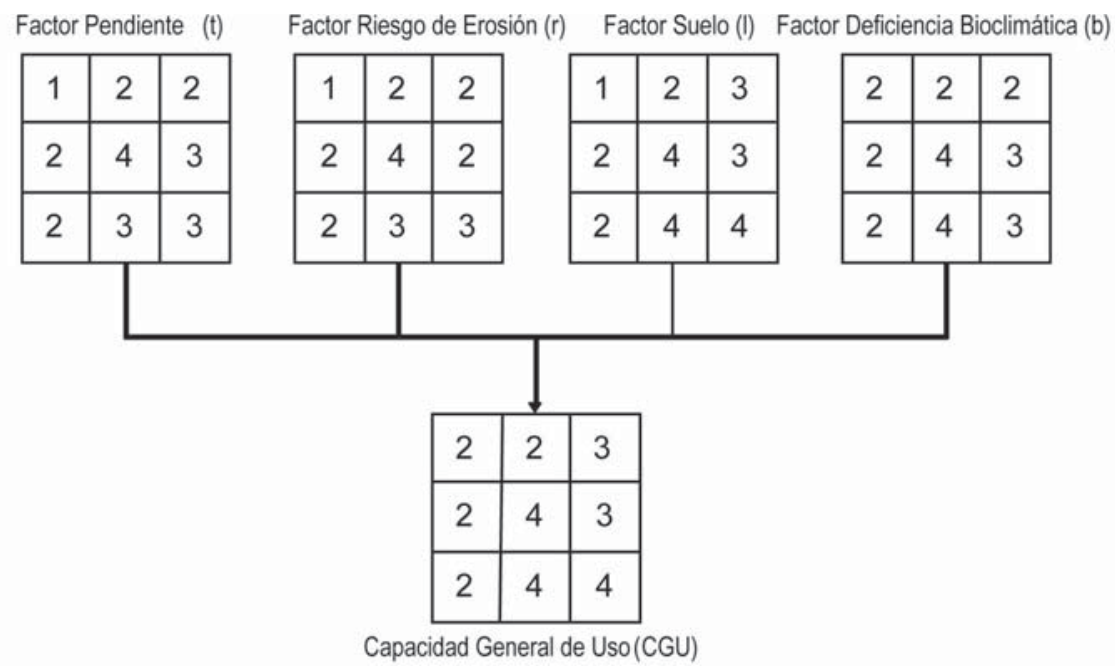

Fuente: elaboración propia.

Por último, a fin de orientar la toma de decisiones en la planificación del uso del suelo, es necesario llevar a cabo una tabulación cruzada entre el mapa CGU y el mapa de uso del suelo, evaluando el grado de adecuación de los usos a la capacidad del territorio. A través del método Delphi (Linstone y Turoff, 1975), un panel multidisciplinario de expertos (geógrafos, geólogos, agrónomos, ingenieros forestales y ecólogos) ha evaluado las combinaciones posibles y se ha reclasificado a cada uno de ellos en una de las siguientes categorías: Áreas Degradadas para ser restauradas, Usos incompatibles, Usos moderadamente incompatibles, Usos compatibles con aplicación de ciertas prácticas de conservación y, por último, los Usos compatibles (tabla 2). 
TABLA 2

MATRIZ RESUMIDA DE LA COMPARACIÓN ENTRE EL MAPA DE CAPACIDAD GENERAL DE USO Y EL MAPA DE USOS DEL SUELO

\begin{tabular}{|c|c|c|}
\hline Clases & $\begin{array}{c}\text { Capacidad General } \\
\text { de Uso }\end{array}$ & Uso del Suelo \\
\hline \multirow{4}{*}{ Áreas de usos compatibles } & $\mathrm{S} 2, \mathrm{~S} 3, \mathrm{~N}$ & Áreas arboladas \\
\hline & $\mathrm{S} 2, \mathrm{~S} 3, \mathrm{~N}$ & Matorrales \\
\hline & S3 & Pastizales \\
\hline & S3 [l y/o b] & $\begin{array}{l}\text { Dehesas, mosaicos de cultivos, } \\
\text { cultivos herbáceos, viñedos y oli- } \\
\text { vares }\end{array}$ \\
\hline $\begin{array}{l}\text { Áreas de usos compatibles } \\
\text { con prácticas de conservación }\end{array}$ & $\mathrm{S} 2$ y/o S3 [t y/o r] & $\begin{array}{l}\text { Dehesas, mosaicos de cultivos, } \\
\text { cultivos herbáceos, viñedos y oli- } \\
\text { vares }\end{array}$ \\
\hline \multirow{3}{*}{$\begin{array}{l}\text { Áreas de usos moderadamente } \\
\text { incompatibles }\end{array}$} & S3 & Cultivos en zonas bajas \\
\hline & $\mathrm{S} 3[\mathrm{t}]$ & Cultivos herbáceos \\
\hline & $\mathrm{N}$ & $\begin{array}{l}\text { Cultivos herbáceos con encinas } \\
\text { dispersas, praderas }\end{array}$ \\
\hline $\begin{array}{l}\text { Áreas de usos altamente } \\
\text { incompatibles }\end{array}$ & $\mathrm{N}$ & Cultivos \\
\hline Áreas degradadas a restaurar & $\mathrm{S} 2, \mathrm{~S} 3, \mathrm{~N}$ & Áreas degradadas \\
\hline Áreas no evaluadas & & Áreas urbanas, zonas rocosas \\
\hline
\end{tabular}

Fuente: elaboración propia a partir de análisis SIG.

\section{RESULTADOS Y DISCUSIÓN}

\section{Capacidad General de Uso de las Tierras}

Los resultados de la evaluación de tierras se expresan en un mapa que contiene las clases y subclases de CGU. Este mapa sintetiza la capacidad general de uso de cada unidad tierra y sus factores limitantes y representa las mejores opciones futuras de los usos agroforestales del suelo para alcanzar un desarrollo sostenible de la tierra. Las manchas con superficies inferiores al tamaño de 
la mínima unidad cartografiable han sido filtradas. En esos casos, las manchas han sido reclasificadas a la clase temática más similar. Los siguientes tipos de usos no han sido evaluados: las zonas urbanas, zonas residenciales, graveras, pantanos y otras áreas artificiales. El mapa de CGU de las tierras (figura 8) muestra la distribución espacial de las tres clases principales ( $\mathrm{S} 2, \mathrm{~S} 3$ y N) y de las 14 subclases presentes en la zona de estudio.

Las tierras de la clase S2 son las que poseen mejor CGU en la zona de estudio. Estas áreas son capaces de soportar el uso agrícola continuo y ocupan una

FIGURA 8

MAPA DE CAPACIDAD GENERAL DE USO DE LAS TIERRAS

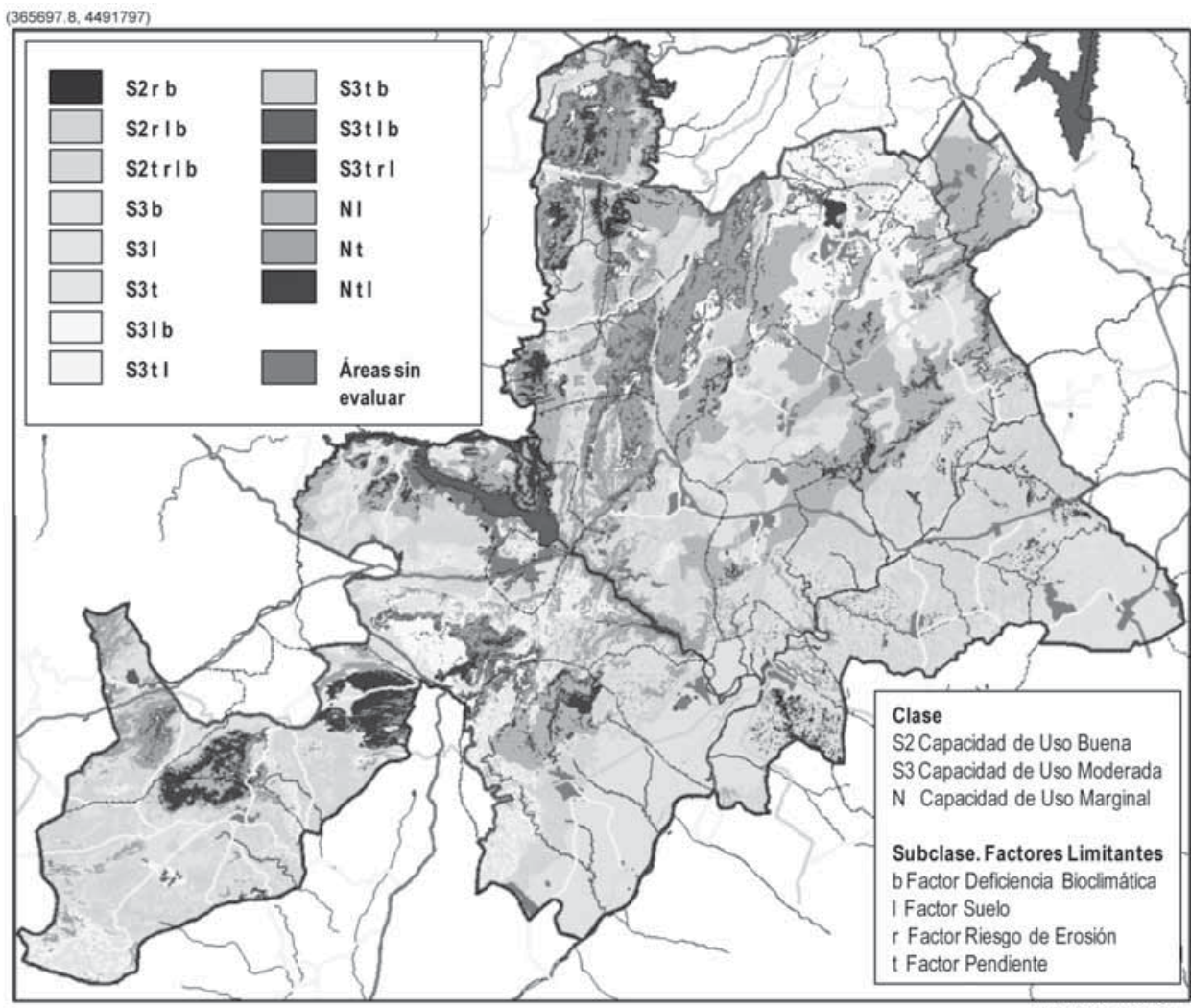

(415657.8, 4450459)

Fuente: elaboración propia. 
superficie de 17.500 hectáreas (21,22\% de toda la ZEPA). Sin embargo, están situadas sobre suelos erodibles y con limitaciones relacionadas con la profundidad útil, su textura, pedregosidad y drenaje. Además, la deficiencia bioclimática, el riesgo de las heladas y la densidad moderada de vegetación son otros factores que limitan el uso de estos suelos. Actualmente, estas tierras soportan los usos tradicionales mediterráneos (cultivos herbáceos, viñas y olivos), aunque algunos de ellos son marginales. La continuidad de estos usos tradicionales debe ser priorizada, ya que éstos son los más adecuados, ecológicamente, a las características biofísicas del terreno. Además, en el caso de los cultivos leñosos, proporcionan mano de obra, durante y después de la cosecha.

Las tierras de clase S3 tienen una capacidad de uso moderado agro-forestal. Esta es la clase más extensa (40.700 ha, aproximadamente el 50\% del territorio) y tiene una amplia combinación de subclases. La clase S3b se caracteriza por problemas de deficiencia bioclimática y ocupa más del 14,5\% de la ZEPA. En algunas zonas, la limitación se debe a los problemas de falta de agua. Este tipo de limitación se ha superado mediante el uso de riego e invernaderos. Un alto riesgo de heladas existe en otras áreas. En otros casos, las limitaciones son el resultado de la escasa profundidad de los suelos (leptosoles y cambisoles dístricos), de drenaje deficiente (cambisoles gléyico) y de las pendientes comprendidas entre el 15 y el 30\%. Estas características biofísicas hacen difícil pensar en una agricultura competitiva. Sería necesaria la introducción de mejoras significativas en las prácticas agrícolas actuales (regadío, uso intensivo de fertilizantes), lo que podría causar, si se introducen, serios impactos negativos sobre un territorio sensible ambientalmente.

Las zonas, comprendidas en esta clase, con los factores más limitantes se aprovechan, principalmente, para usos forestales o ganaderos. Como consecuencia de su carácter multifuncional, estas áreas podrían cumplir una tarea importante en la diversificación de la economía local. Dichas funciones incluyen el aprovechamiento económico de productos tradicionales como el piñón, la madera, bayas, setas y otros hongos, junto a otras funciones como las recreativas, la protección ecológica y ambiental (control de la erosión, la regulación del ciclo del agua, fijación de carbono, y la creación de suelo). La presencia de ganado caprino y ovino controlado en las zonas forestales es una muestra de la diversidad hacia la que se dirigen las tierras rurales de la UE, con baja productividad y problemas estructurales. Sin embargo, estos usos tradicionales han demostrado ser ambientalmente sostenibles, dado que son más adecuados a la capacidad general de uso de las tierras sobre las que se asientan.

La clase N abarca todas las áreas con peor CGU. Sus características biofísicas son muy limitadas para soportar cultivos agrícolas y otros usos agrofores- 
tales intensivos. Pendientes superiores al 30\% y los suelos con menores profundidades útiles (leptosoles dístricos y líticos) explican la productividad marginal de estas tierras. Estas tierras son las más utilizadas con fines ganaderos o forestales y, a pesar de su baja capacidad de uso, merecen una protección especial desde el punto de vista ecológico. Cubren 21.000 ha (26\% del territorio). A pesar de su escasa aptitud de uso están localizadas sobre lugares de elevada belleza escénica, alta calidad visual del paisaje y gran valor ecológico. Estas áreas tienen cualidades recreativas, educativas y ambientales, por lo que pueden estimular el desarrollo local a través del turismo rural, junto con otros activos como el patrimonio histórico y cultural y la gastronomía.

\section{Adaptación del uso del suelo a la CGU}

Una tabulación cruzada entre el mapa de uso del suelo y el modelo CGU ofrece información relativa al grado de adecuación de las actividades humanas al entorno físico sobre el que se asientan. En resumen, se comparan los usos actuales con los usos potenciales. Ésta es una información estratégica para el planificador y el gestor de esta área protegida.

El resultado de la tabulación cruzada es un mapa de idoneidad de usos (figura 9). La leyenda muestra las siguientes categorías:

1. Las áreas de usos compatibles ocupan la mayor parte de la ZEPA. Se distribuyen sobre una superficie de 63.109 ha (76\% del territorio). Este grupo incluye áreas de bosque, matorrales, pastizales, dehesas cultivadas, cultivos herbáceos y leñosos sobre suelos de escasa capacidad de uso, principalmente S31, S3b y S3lb. De acuerdo a recientes trabajos predictivos, en los que se simulan varios escenarios futuros para toda la Comunidad de Madrid (Gallardo y Martínez-Vega, 2012) y para la ZEPA «Encinares del Río Alberche y Cofio» (Millington et al. 2007, 2008, 2009 y 2011), se prevé que estas áreas se incrementen, aún más, como consecuencia del abandono de las tierras agrícolas marginales.

2. Las áreas de usos compatibles con prácticas de conservación ocupan 10.098 ha, el 12,18\% del área de estudio. Este grupo está formado por cultivos herbáceos y leñosos, las dehesas y los pastos cultivados en suelos con una capacidad de uso media o buena. Estas áreas, generalmente, muestran una buena adaptación entre los usos y los factores físicos, aunque tienen ciertas limitaciones: pendiente y riesgo de erosión. Estas 
combinaciones deben ser tomadas en cuenta con el fin de evitar que ciertas actividades inapropiadas generen pérdidas de suelo y, a largo plazo, el deterioro de estas áreas. Es conveniente adoptar buenas prácticas agrarias para garantizar su conservación.

3. Las áreas de usos moderadamente incompatibles se extienden a lo largo de 984 ha (1,19\%). Este grupo incluye a los cultivos herbáceos sobre suelos con una CGU media, así como a los cultivos herbáceos y pastos con encinas dispersas (paisajes adehesados) sobre suelos con capacidad

FIGURA 9

\section{MAPA DE IDONEIDAD DE USOS}

$(365697.8,4491797)$

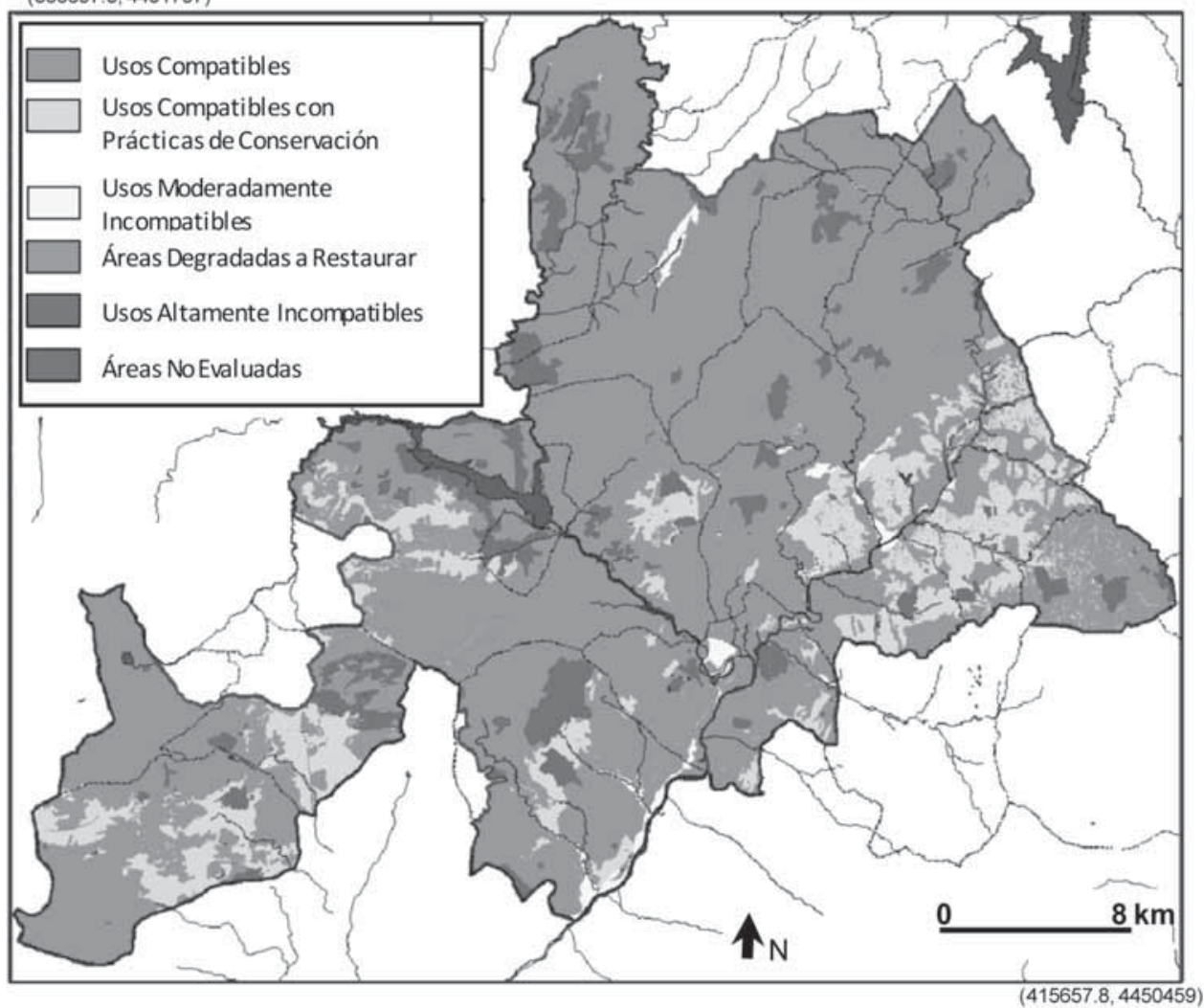

Fuente: elaboración propia.

Estudios Geográficos, Vol. LXXIII, 273, pp. 551-580, julio-diciembre 2012

ISSN: 0014-1496, eISSN: 1988-8546, doi: 10.3989/estgeogr.201219 
de uso marginal. Estas áreas tienen, en general, un rendimiento medio y una baja productividad como resultado de los numerosos factores limitantes. Sin embargo, tienen la ventaja de su multifuncionalidad (coexistencia de usos agrícolas, ganaderos y cinegéticos), que les aporta un valor añadido desde el punto de vista ambiental.

4. Las áreas degradadas a restaurar se extienden a través de 1.304 ha ( $1,57 \%$ de la ZEPA). Este grupo incluye todas las áreas degradadas, como las que han sido afectadas por incendios forestales a lo largo de los últimos años, así como las tierras agrícolas abandonadas. La mayoría de estas tierras tienen una capacidad biofísica de uso regular o buena, aunque se encuentran afectadas por problemas estructurales o circunstanciales, lo que ha ocasionado su degradación o abandono. El planificador debe tratar de organizar la actividad humana en estas áreas, la restauración de tierras degradadas, la preservación de las zonas con mejores cualidades, y la asignación de nuevos usos de acuerdo a su capacidad.

5. Las áreas de usos altamente incompatibles ocupan 414 ha $(0,5 \%$ del área protegida). La mayoría de estas áreas son pequeñas parcelas de cultivos herbáceos y leñosos (olivos y viñedos) con una capacidad de uso agroforestal marginal (clase N). Sin lugar a dudas, estos espacios serán eventualmente abandonados, debido a su baja productividad y a la dificultad de cultivar sobre ellos.

En resumen, la aplicación tradicional de buenas prácticas agrícolas, usos de la tierra de baja intensidad y una adaptación de los usos a las condiciones físicas del territorio, han hecho posible la preservación de los recursos naturales, pudiendo legarlos a las generaciones siguientes. A pesar de esta tendencia general positiva, existen pequeñas áreas en las que se identifica falta de concordancia entre los usos actuales y los usos potenciales. Es en estas áreas donde el planificador debe poner especial énfasis para mejorar la sostenibilidad del sistema. Por otra parte, como se mencionó en la descripción del área de estudio, la proximidad de este territorio al Área Metropolitana de Madrid amenaza con provocar un desarrollo incontrolado si los planes de gestión sostenible no se aplican, adecuadamente, o si se aprueban, irregularmente, los planes urbanísticos de los municipios incluidos en la ZEPA, tal como han venido denunciando, en los últimos años, organizaciones como Ecologistas en Acción (2006). 


\section{CONCLUSIONES}

Tecnológicamente, la integración de las rutinas de un sistema experto de evaluación de tierras, como MicroLEIS, en el entorno de un SIG y el empleo de un MDE ha producido una mejora en los resultados obtenidos, si se compara con otras soluciones empleadas en otros trabajos similares. Metodológicamente, la consideración de la capacidad general de uso de las tierras se recomienda en la planificación del uso del suelo, con el fin de mejorar la gestión sostenible del territorio. A pesar de las dificultades en su aplicación práctica, la evaluación de tierras es una herramienta muy ventajosa, lo que facilita el proceso de toma de decisiones y la selección de los usos más adecuados de acuerdo a los objetivos de planificación (de la Rosa et al., 2009).

Los resultados demuestran que la población local ha logrado preservar los recursos naturales excepcionales de este territorio a lo largo de la historia, por medio de los usos tradicionales sostenibles. Sin embargo, el abandono de las funciones tradicionales de los espacios agroforestales y la proliferación de nuevos usos, infraestructuras y actividades de ocio al aire libre pueden alterar el equilibrio ecológico. Algunas de estas transformaciones están teniendo un impacto negativo sobre los ecosistemas fundamentales, especialmente preocupante en lo que se refiere a la fragmentación de hábitats prioritarios.

El método de evaluación de tierras, junto con otros métodos (Arroyo-Mora et al., 2005), debe contribuir a la planificación armoniosa de los usos del suelo que garantiza la conservación de los recursos naturales de mayor valor en las áreas protegidas.

Fecha de recepción: 05/09/2012

Fecha de aceptación: 16/10/2012

\section{BiBLIOgRAFíA}

Aldama, J. J. (1996): Actuaciones para la conservación del Lince Ibérico en la Comunidad de Madrid. Madrid, Consejería de Medio Ambiente y Desarrollo Regional.

Álvarez, M. y Díaz, J. (1997): "Seguimiento y conservación de águilas reales y perdiceras en el Sistema Central". Quercus, 141, pp. 18-22.

Aronoff, S. (1989): Geographic information systems: a management perspective. Ottawa, WDL Publications.

Arroyo-Mora, J. P.; Sanchez-Azofeifa, G. A.; Rivard, B.; Calvo, J. C. y Janzen, D. H. (2005): "Dynamics in landscape structure and composition for the Chorotega re- 
gion, Costa Rica from 1960 to 2000”. Agriculture, Ecosystems E Environment, 106/1, pp. 27-39.

Blondel, J. y Aronson, J. (1999): Biology and Wildlife of the Mediterranean Region. Oxford, Oxford University Press.

Bronsveld, K.; Huizing, H. y Omakupt, M. (1994): "Improving land evaluation and land use planning". ITC Journal, 1994/4, pp. 359-365.

Caraveli, H. (2000): "A comparative analysis on intensification and extensification in Mediterranean agriculture: dilemmas for LFAs policy". Journal of Rural Studies, 16, pp. 231-242.

Chrisman, N. R. (1989): "Modelling error in overlaid categorical maps", en M. F. Goodchild y S. Gopal (eds.): Accuracy of spatial databases. London, Taylor \& Francis, pp. 21-34.

Chuvieco, E.; Bosque, J. y Salas, F. J. (1991): "An evaluation of interpolation methods to generation elevation data”, en D. A. Ondaatje (ed.): EGIS'91 Proceedings, Utrecht, EGIS Foundation, p. 1309.

Chuvieco, E.; Aguado, I.; Yebra, M.; Nieto, H.; Salas, J.; Martín, M. P.; Vilar, L.; Martínez-Vega, J.; Martín, S.; Ibarra, P.; de la Riva, J.; Baeza, J.; Rodríguez, F; Molina, J. R.; Herrera, M. A. y Zamora, R. (2010): "Development of a framework for fire danger assessment using Remote Sensing and Geographic Information System technologies". Ecological Modelling, 221, pp. 46-58.

Consejería de Medio Ambiente y Desarrollo Regional de la Comunidad de Madrid, (1998): Plan Forestal de la Comunidad de Madrid: Programa de protección y manejo de la fauna. Madrid, Consejería de Medio Ambiente y Desarrollo Regional de la Comunidad de Madrid.

Cowling, R. W.; Rundel, P. W.; Lamont, B. B.; Arroyo, M. K. y Arianoutou, M. (1996): "Plant diversity in Mediterranean-climate regions". Trends in Ecology and Evolution, 11, pp. 362-366.

Eastman, J. R. (2000): "Decision Strategies in GIS”. Directions Magazine. Disponible en: http://www.directionsmag.com. (Fecha de consulta: 23/10/2012).

Ecologistas en Acción (2006): Análisis del borrador del PORN de la ZEPA "Encinares del río Cofio y río Alberche". Estudio comparativo entre el borrador de 2001 y el borrador de 2005. 30 pp. Disponible en: http://www.ecologistasenaccion.org/IMG/pdf/In forme_PORN_ZEPA-Suroeste.pdf./Fecha de consulta: 16/10/2012).

FAO (1976): “A Framework for Land Evaluation", en International Institute for Land Reclamation and Improvement (ed.): Soil Bulletin, 32. Rome, Development and Conservation Service.

FAO-UNESCO (1989): Mapa mundial de suelos. Leyenda revisada. Roma, FAO.

Flores, P.; Martínez de Anguita, P.; Romero-Calcerrada, R.; Novillo, C. y Ruiz, M. A. (2008): "Los sistemas de pago por servicios ambientales entre la adicionalidad y la subsidiariedad: aplicación a la belleza escénica en el pantano de San Juan, Madrid, España". Investigación Agraria: Sistemas y Recursos Forestales, 17/1, pp. 39-53. 
Frolov, Y. S. y Maling, D. H. (1969): "The accuracy of area measurements by point counting techniques". Cartographic Journal, 6, pp. 21-35.

Gallardo, M. y Martínez-Vega, J. (2012): "Cambios de usos del suelo en la Comunidad de Madrid: analizando el pasado y simulando el futuro", en J. Martínez-Vega y M. P. Martín Isabel (eds.): Tecnologías de la Información Geográfica en el contexto del Cambio Global. Madrid, CSIC-AGE, pp. 305-314.

García González, F. (ed.) (1998): Control, seguimiento y marcaje de la nutria en la Comunidad de Madrid. Año 1998. Informe inédito. Madrid, Consejería de Medio Ambiente y Desarrollo Regional.

García Perera, R. (2000): "Survival of injured Iberian Lynx (Lynx pardinus) and nonnatural mortality in central-southern Spain". Biological Conservation, 93, pp. 265-269.

Garzón, J.; González, L. M.; González, J. L. y Hiraldo, E. (1984): "Situación actual y problemática del Águila Imperial Ibérica (Aquila adalberti)”. Rapinyaires Mediterranis II, pp. 70-80.

González Bernáldez, F. (1991): "Ecological consequences of the abandonment of tradicional land-use systems in Central Spain". Options Méditerranéenes, Série Séminaires 15 , pp. 23-29.

Hommel, P. W. F. (1990): "Ujung Kulon: landscape survey and land evaluation as a habitat for the Javan rhinoceros". ITC Journal, 1990-1, pp. 1-15.

ICONA (1988): Agresividad de la lluvia en España. Valores del factor R de la Ecuación Universal de Pérdidas de Suelo, Madrid, Ministerio de Agricultura, Pesca y Alimentación.

Intergraph (1994): MGE Terrain Analyst. User's guide for the Windows NT Operating System, Hunstville, AL.

Kidner, D. y Jones, C. (1991): "Implicit triangulation for large terrain databases". en D. A. Ondaatje (ed.): EGIS'91 Proceedings, Utrecht, EGIS Foundation, pp. 537-546.

Lamelas, M. T.; Hoppe, A.; de la Riva, J. y Marinoni, O. (2009): "Modelling environmental variables for geohazards and georesources assessment to support sustainable land-use decisions in Zaragoza (Spain)". Geomorphology, 111, pp. 88-103.

Linstone, H. A. y Turoff, M. (eds.) (1975): The Delphi Method. Techniques and Applications. Reading, Addison-Wesley.

MacDonald, D.; Crabtree, J. R.; Wiesinger, G.; Dax, T.; Stamou, N.; Fleury, P.; Gutierrez-Lazpita, J. y Gibon, A. (2000): "Agricultural abandonment in mountain areas of Europe: environmental consequences and policy response". Journal of Environmental Management, 59, pp. 47-69.

Machin, J. y Navas, A. (1995): "Land evaluation and conservation of semiarid agrosystems in Zaragoza (NE Spain) using an expert evaluation system and GIS". Land Degradation and Rehabilitation, 6/4, pp. 203-214.

Martínez-Vega, J. y Martín Lou, M. A. (coords.) (2000): Proyecto de Plan de Ordenación de Recursos Naturales de la Zona de Especial Protección para las Aves $n^{\circ} 56$ "Encinares de los ríos Alberche y Cofio". Madrid, CSIC, 301 pp. 
Martínez-Vega, J.; Martín, M. P.; Romero-Calcerrada, R.; Martínez, J. y Echavarría, P. (2003): "Aplicación de los SIG a los modelos de riesgo de incendios forestales: riesgo humano a escala regional", en J. L. Gurría (ed.): IX Conferencia Iberoamericana de SIG. Cáceres, Universidad de Extremadura.

Millington, J. D. A.; Perry, G. L. W. y Romero-Calcerrada, R. (2007): "Regression techniques for examining land use/cover change: A case study of a mediterranean landscape". Ecosystems, 10/4, pp. 562-578.

Millington, J.; Romero-Calcerrada, R.; Wainwright, J. y Perry, G. (2008): “An agentbased model of mediterranean agricultural land-use/cover change for examining wildfire risk". JASSS, 11/4.

Millington, J. D. A.; Wainwright, J.; Perry, G. L. W.; Romero-Calcerrada, R. y Malamud, B. D. (2009): "Modelling Mediterranean landscape succession-disturbance dynamics: A landscape fire-succession model". Environmental Modelling \& Software, 24/10, pp. 1196-1208.

Millington, J. D. A.; Demeritt, D. y Romero-Calcerrada, R. (2011): "Participatory evaluation of agent-based land-use models". Journal of Land Use Science, 6/2-3, pp. 195-210.

Navas, A.; Machín, J. y Soto, J. (2005): “Assessing soil erosion in a Pyrenean mountain catchment using GIS and fallout ${ }^{137} \mathrm{Cs".} \mathrm{Agriculture,} \mathrm{Ecosystems} \mathrm{and} \mathrm{Environ-}$ ment, 105, pp. 493-506.

Pérez-Hugalde, C.; Romero-Calcerrada, R.; Delgado-Pérez, P. y Novillo, C. J. (2011): "Understanding land cover change in a Special Protection Area in Central Spain through the enhanced land cover transition matrix and a related new approach". Journal of Environmental Management, 92/4, pp. 1128-1137.

Peucker, T. K.; Fowler, R. J.; Little, J. J. y Mark, D. M. (1978): "The triangulated irregular network". Proceedings of the DTM Symp, St. Louis (MI), American Society of Photogrammetry-American Congress on Survey and Mapping, pp. 24-31.

Pinto-Correia, T. (2000): "Future development in Portuguese rural areas: how to manage agricultural support for landscape conservation?". Landscape and Urban Planning, 50, pp. 95-106.

Romero-Calcerrada, R. (2000): La valoración socioeconómica en la planificación de espacios singulares: Las Zonas de Especial Protección de Aves. Alcalá de Henares, Universidad de Alcalá, 331 pp.

Romero-Calcerrada, R. (2002): "Metodología para la planificación y desarrollo sostenible en Espacios naturales Protegidos europeos: las Zonas de Especial protección para las Aves". Geofocus, 2, pp. 1-32.

Romero-Calcerrada, R. y Martínez-Vega, J. (2001): "La cartographie climatique dans la planification des Zones de Protection Spéciale d'Oiseaux", en M. F. Pita (ed.): Climat et environnement. Linformation climatique au service de la gestion de l'environnement, Sevilla, Association International de Climatologie and University of Sevilla.

Romero-Calcerrada, R. y Perry, G. L. W. (2002): "Landscape change pattern (19841999) and implications for fire incidence in the SPA Encinares del rio Alberche y 
Cofio (Central Spain)", en D. X. Viegas (ed.): Forest Fire Research E Wildland Fire Safety. Rotterdam, Millpress.

Romero-Calcerrada, R. y Perry, G. L. W. (2004): "The Role of Land Abandonment in Landscape Dynamics in the SPA 'Encinares del río Alberche y Cofio' Central Spain, 1984-1999". Landscape and Urban Planning, 66/4, pp. 217-232.

Romero-Calcerrada, R.; Barrio-Parra, F; Millington, J. D. A., y Novillo, C. J. (2010): "Spatial modelling of socioeconomic data to understand patterns of human-caused wildfire ignition risk in the SW of Madrid (central Spain)". Ecological Modelling, 221/1, pp. 34-45.

Rosa, D. de la; Moreno, J. A.; García, L. V. y Almorza, J. (1992): "MicroLEIS: A microcomputer-based Mediterranean land evaluation information system". Soil Use and Management, 8, pp. 89-96.

Rosa, D. de la ; Mayol F; Diaz-Pereira, E.; Fernandez, M. y Rosa Jr., D. de la (2004): “A land evaluation decisión support system (MicroLEIS DSS) for agricultural soil protection with special reference to the Mediterranean region". Environmental Modelling E Software, 19, pp. 929-942.

Rosa, D. de la; Anaya-Romero, M.; Diaz-Pereira, E.; Heredia, N. y Shahbazi, F. (2009): "Soil-specific agro-ecological strategies for sustainable land use. A case study by using MicroLEIS DSS in Sevilla Province (Spain)". Land Use Policy, 26, pp. 10551065.

Rossiter, D. G. y van Wambeke, A. R. (1989): Automated Land Evaluation System (ALES). Version 2. Ithaca, Cornell University.

Saalfeld, A. (1987): "Stability of map topology and robustness of map geometry". AutoCarto8 Proceedings, Baltimore, pp. 78-86.

Schultink, G. (1987): "The CRIES resource information system: computer-aided spatial analysis of resource development potential and development policy alternatives". en K. J. Beek; P. A. Burrough y D. E. McCormack (eds.): Quantified Land Evaluation Procedures. Enschede, ITC, pp. 95-99.

Shahbazi, F. y Jafarzadeh, A. A. (2010): "Integrated assessment of rural lands for sustainable development using MicroLEIS DSS in West Azerbaijan, Iran". Geoderma, 157, pp. 175-184.

Skidmore, A. K. (1989): "A comparison of techniques for calculating gradient and aspect from a gridded digital elevation model”. International Journal of Geographical Information Systems, 3/4, pp. 323-334.

Stoate, C.; Boatman, N. D.; Borralho, R. J.; Rio Carvalho, C.; Snoo, G. R. de y Eden, P. (2001): "Ecological impacts of arable intensification in Europe". Journal of Environmental Management, 63, pp. 337-365.

Thornthwaite, C. W. (1948): "An approach toward a rational classification of climate". The Geographical Review, 38, pp. 55-94.

Uboldi, J. A. y Chuvieco, E. (1997): "Using remote sensing and GIS to assess current land management in the valley of the Colorado river, Argentina". ITC Journal, 1997/2, pp. 160-165. 
USDA (1961): Land Capability classification. Agricultural Handbook, 210. Washington, SCS.

Veiga, J. P.; Alonso, J. C. y Alonso, J. A. (1984): "Sobre la población de Águilas imperiales (Aquila heliaca adalberti) de la Sierra de Guadarrama". Rapinyaires Mediterranis, II, pp. 54-59.

Verheye, W. H. (1987): "Quantified land evaluation as a basis for alternative land use planning”, en K. J. Beek, P. A. Burrough y D. E. McCormack (eds.): Quantified Land Evaluation Procedures. Enschede, ITC, pp. 144-146.

Wood, S. R. y Dent, F. J. (1983): LECS a Land Evaluation Computer System Methodology. Bogor, Centre for Soil Research, Ministry of Agriculture, Government of Indonesia.

Zuviría, M. de y Valenzuela, C. R. (1994): "Mapping land suitability for coffee with ILWIS". ITC Journal, 1994/3, pp. 301-307.

\section{RESUMEN}

Se ha utilizado un sistema experto de Evaluación de Tierras, integrado en un Sistema de Información Geográfica, para evaluar la capacidad general de uso de las tierras y compararla con los usos actuales del suelo en un área protegida. El valle medio del río Alberche, situado en el suroeste de la región de Madrid (centro de España), ha sido declarado Zona de Especial Protección para las Aves y forma parte de la red Natura 2000. Se han identificado zonas de conflicto entre el desarrollo y la conservación de la naturaleza. Los resultados ayudan a orientar las políticas públicas en materia de conservación de los recursos naturales de la zona y a planificar el uso futuro de la tierra en el contexto del desarrollo sostenible.

PAlabras Clave: evaluación de tierras; cartografía de ocupación y usos del suelo; Zona de Especial Protección para las Aves (ZEPA); Sistema de Información Geográfica (SIG); río Alberche; Madrid; España.

\section{Abstract}

We used an expert system for land evaluation, integrated into a Geographic Information System to assess the overall capacity of land use and to compare it with the current land uses in a protected area. The mid valley of the Alberche river, located in the southwest region of Madrid (central Spain), has been declared a Special Protection Area and is part of the Natura 2000 network. Conflict zones have been identified among the development and the conservation of the nature. The results will help to drive the public policies regarding conservation of the zone natural resources and to plan the future land use in the context of sustainable development.

KEY WORDS: land evaluation; Land Cover-Land Use mapping; Special Protection Areas (SPA); Geographical Information System (GIS); Alberche river; Madrid; Spain. 


\section{RÉSUMÉ}

Nous avons utilisé un système expert pour l'évaluation des terres, intégrés dans un système d'information géographique pour évaluer la capacité globale de l'utilisation des terres et de la comparer avec la terre actuelle utilise dans une zone protégée. La vallée de la rivière Alberche, situé dans la région sud-ouest de Madrid (centre de l'Espagne), a été déclarée zone de protection spéciale pour les oiseaux et fait partie du réseau Natura 2000. Ont été identifiées les zones de conflit entre développement et conservation. Les résultats permettent d'orienter les politiques publiques sur la conservation des ressources naturelles dans la région et un plan pour utilisation future des terres dans le contexte du développement durable.

Mots CLÉs: évaluation des terres; cartographie de l'occupation et de l'utilisation des sols; Zone de Protection Spéciale pour les Oiseaux (ZPS); Système d'Information Géographique (SIG); Alberche; Madrid; Espagne. 